\title{
A METHOD FOR TECHNICAL DIAGNOSIS OF CONSTRUCTION
}

\author{
NguYen Van Pho \\ Hanoi University of Civil Engineering
}

\section{§1. Introduction}

Nowadays the design of a new construction is no more a hard problem, but it is not the same to the technical diagnosis problem of constructions.

The methods, largely used now for the diagnosis problem of construction generally include the following steps:

- To carry out inspection from the real construction for gathering the needed data by investigations, measurements, experiments, etc.

- To model the real construction (scheme of the calculation).

- To set up the system of equations for diagnosis.

- To solve above the system of equations.

- To evaluate and conclusion.

The essence of the above procedure is to solve an inverse (or partly inverse) problem of mechanics $[1,2,3]$, therefore the following difficulties often rise: 1

- The system of equations is non-linear or transcendent.

- The system of equations of diagnosis has more than one solution (the number of solutions is finite).

- The solutions of system may be unstable due to errors of experimental data.

- The system has no solution or an infinity of solutions.

Hence up to now there is no yet any program to auspiciously solve the diagnosis problem in the computer, like SAP90 program does for solving the problem to design a new construction.

In the present article the author advances a diagnosis method of structures different from the above, we expect that the advanced method could avoid some dificulties inherent with the solution of inverse problem.

The matter of our method is to solve a sequence of design problems, and to male a comparison between the obtained results so as to deduce the corresponding inverse problem. The similar idea is used in $[4,5]$. 
For illustration, diagnostic problems of circular and rectangular plates are considered.

$\S 2$. The contents of the method

The method includes following steps:

Step 1: Defining the diagnosis task.

Express the unknowns of the diagnosis problem by a vector

$$
\vec{k}=\left\{k_{1}, k_{2}, \ldots, k_{n}\right\}^{T}
$$

The elements $k_{i}(i=1,2, \ldots, n)$ are generally geometrical or physical characteristics of the construction, in some case they can be chosen as kinetic and dynamic characteristics.

Step 2: Gathering data for diagnosis.

Let the set of data, obtained by inspection be

$$
\vec{\delta}_{0}=\left\{\delta_{1}^{(0)}, \delta_{2}^{(0)}, \ldots, \delta_{m}^{(0)}\right\}
$$

The data are generally the responses of the construction, such as stresses, strains, deformations, frequencies, amplitudes, etc.

Step 3: Setting up and solving diagnostic system of equations.

1. The system of equations for diagnosis is set up according to the methods of structural mechanics, for example the finite element method [6].

a. Case of static problem.

$$
\boldsymbol{A X}=\boldsymbol{B}
$$

b. Case of dynamic problem

$$
M \ddot{X}+C \dot{X}+k X=B .
$$

2. By preliminary inspection and by referring to the design's documents, one can determine the upper and the lower bounds of each $k_{i}$.

$$
a_{i} \leq k_{i} \leq b_{i} \quad(i=1,2, \ldots, n)
$$

where $a_{i}, b_{i}$ are constants.

Thus the diagnosis variables $k_{i}$ lay in the interior or on the boundary of a convex, closed, bounded hyperdomain $\bar{G}$, which is called the domain of possible values. 
3. One discretizes $\bar{G}$ by creating on it a finite set of points $\overline{G_{h}}, h$-a parameter of discretization. Now, instead of considering the points $\vec{k}=\left(k_{1}, k_{2}, \ldots, k_{n}\right)$ in the whole $\bar{G}$, one considers them only in the finite set of points

$$
\vec{k}^{(j)}=\left\{k_{i}^{(j)}\right\} \quad(j=1,2, \ldots, p),
$$

which constitute $\bar{G}_{h}$. Here $j$ means an index of numeration of points on $\bar{G}_{h}$.

4. One puts the values (2.6) into the system (2.3) or (2.4), then the diagnostic system of equations becomes a system of equations of a design problem. Solving the latter system of equations, one gets the values

$$
\vec{\delta}=\left\{\delta_{i}\right\} \quad(i=1,2, \ldots, m)
$$

Step 4: Finding the solution of the diagnosis problem.

Now one considers that the set of computed values (2.7) is an approximate representation of the $\vec{\delta}_{0}$ set (2.2) of experimental data. From this one proceeds find the solution of the diagnosis problem as follows:

1. Compute the dispersions $\Delta_{j}$ between $\vec{\delta}_{0}$ and $\vec{\delta}^{(j)}$

$$
\Delta_{j}=\left\{\sum_{i=1}^{m}\left(\delta_{i}^{(j)}-\delta_{i}^{(0)}\right)^{2}\right\}^{\frac{1}{2}}
$$

here the index $j$ runs along all the points of $\bar{G}_{h}$

2. Find

$$
\Delta_{s}=\min _{(j)} \Delta_{j}
$$

According to the degree of approximation, one seeks in advance a value $\Delta_{0}$. If

$$
\Delta_{8} \leq \Delta_{0}
$$

then one takes the value of $\vec{\delta}$ at the point $S$

$$
\vec{\delta}_{s}=\left\{\delta_{1}, \delta_{2}, \ldots, \delta_{m}\right\}_{s}
$$

as the solution of the diagnosis problem.

From this values, we deduce values of $\left(k_{1}, k_{2}, \ldots, k_{m}\right)_{s}$, it is the solution of the diagnosis problem.

Remarks : The procedure described above to solve the diagnosis problem satisfies two requirements:

a. The solution obtained the best approximation of the experimental data. 
b. This solution satisfies the system of equations of structural mechanics. But the procedure has two shortcomings:

a. The number of points of $\bar{G}_{h}$ being too high, the solution requires an enormous volume of computation.

b. The reliability or the degree of precision of the method, has not yet been analyzed.

These questions will discussed in following paragraph.

$\S 3$. Reduction of the computation by using the algorithm of solving optimization problems

In the optimum experimental theory [7], it is well known that to accelerate getting the optimal solution it is not necessary to check all the admissible plans. An illustration of this idea could be given in the simple case of two dimensional diagnosis problem is being solved by the method of $\$ 2$.

The variables are the characteristics $E, \nu$ of an elastic material, which have boundaries.

$$
\begin{aligned}
& a_{1} \leq E \leq b_{1} \\
& a_{2} \leq \nu \leq b_{2}
\end{aligned}
$$

Fig 1. represents the discretized domain $\bar{G}_{h}$, on which the dispersions $\Delta_{j}(j=$ $1,2,3,4,5)$ and the $\Delta_{s}$ on $(2.8),(2.9)$ are computed. Suppose that

$$
\min _{(j=1,2,3,4,5)} \Delta_{j}=\Delta_{s}=\Delta_{2}
$$

Likewise, let

$$
\min _{(j=1,2,6,7,8)} \Delta_{j}=\Delta_{7}
$$

Thus, the itinerary has gone from point 1 to point 2 , then from point 2 to point 7 , still so doing, $\Delta_{s}$ will be found not with standing every point. Therefore, the computation volume is reduced clearly.

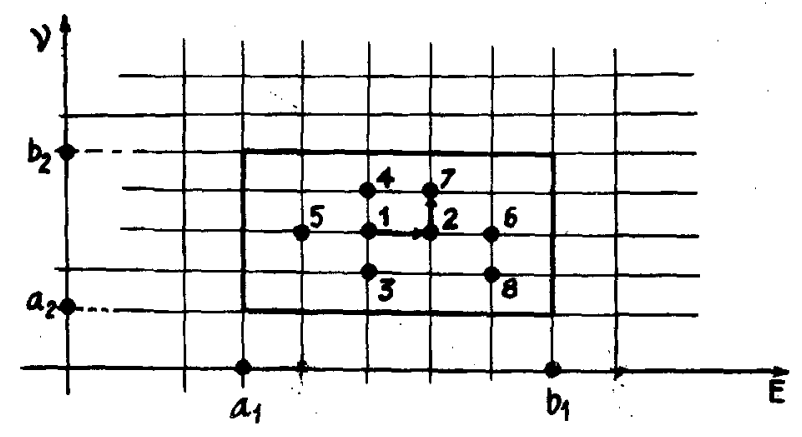

Fig. 1 


\section{\$4. On the exactness of the diagnosis method}

In the technical diagnosis problem the degree of precision of a solution depends not only upon the method of diagnosis, yet it still depends mainly upon the quantity and quality of the informations obtained from the real system. We don't consider the precision degree concerned with the modeling of the real construction ( $\mathrm{i}$ - e the choice of the computed scheme), because it is a common question to every method.

As regards the best approximation of measurement's data, it first depends upon the choice of $\Delta_{0}$, however the most important purpose here is the quality and quantity of informations.

The observation and measurement's data must reflect the essential features of the real system.

For example, if the displacements are chosen as measurement's data, then the configuration features of the system must be well reflected. If the state parameters of the system depend upon space and time. then the measurement's data must be taken into account with space and time. The more the quantity and quality about the real system are greater, the degree of precision of diagnosis is higher, but the informations have to be independent.

Note that if the informations are gathered sufficiently in quantity and quality, then there is no need to solve the diagnosis problem, because all the unknowns are really in the experimental results.

However in the real structures there are informations not attainable. For example, dimensions or defects of piles in the earth. In these cases the recourse to the diagnosis problem is needed.

It is also just by these reasons that the solution of the diagnosis problem is precise only to a certain degree.

§5. The diagnosis problem of the characteristics values of materials via the measurement data of displacements

As an illustration of the method presented above, the author consider below the diagnosis problem according to the measurement's data of displacement at some points of the construction.

Generally to diagnosis the construction one can use different categories of informations by provoking actions and getting responses from the real system. The actions to the system can be static or dynamic.

Because of the means to gather informations, the requirements to secure the construction and other requirements of the construction's owner etc, some ways to get information like provoking impulse, explosion, vibration etc, are not available. In these cases one can put static loads and measure the displacements to make 
diagnosis. Obviously the diagnosis method by measuring the displacements at some points of structure is not available in certain cases, such as diagnosis the defects of piles or the quantity of a deep foundation.

Now we first consider some simple cases.

1. Case of a simply supported beam of length $a$ is subjected to uniformly distributed load of intensity $q$.

Suppose that we have got measurement

$$
y_{\max }=y\left(\frac{a}{2}\right)=y_{0}
$$

From the solution of the elastic curve equation, we have

$$
y\left(\frac{a}{2}\right)=y_{\max }=\frac{5 q a^{2}}{384 E J}
$$
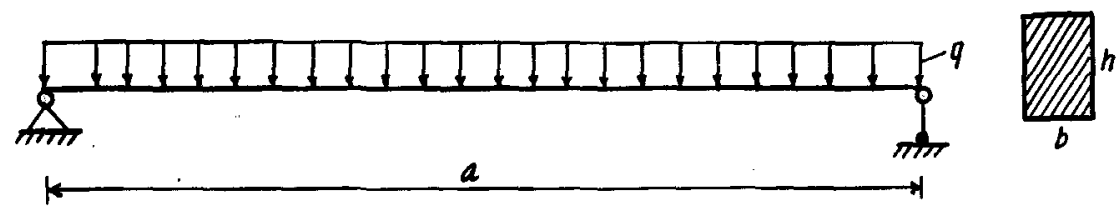

Fig. 2

If $q, a$ and $y_{0}$ are got and the value to diagnose is the stiffness of the beam $E J$, then

$$
E J=\frac{5 q a^{4}}{384 y_{0}} .
$$

Let the errors of $q, a$ and $y_{0}$ be $\varepsilon_{q} \geq 0, \varepsilon_{a} \geq 0, \varepsilon_{y_{0}} \geq 0$, then

$$
E J=\frac{5}{384}\left[\frac{\left(q \pm \varepsilon_{q}\right)}{\left(y_{0} \pm \varepsilon_{y_{0}}\right)}\left(a \pm \varepsilon_{a}\right)^{4}\right]
$$

then we deduce $A \leq E J \leq B$ where:

$$
\begin{aligned}
& A=\frac{5}{384}\left[\frac{\left(q-\varepsilon_{q}\right)}{\left(y_{0}+\varepsilon_{y_{0}}\right)}\left(a-\varepsilon_{a}\right)^{4}\right] \\
& B=\frac{5}{384}\left[\frac{\left(q+\varepsilon_{q}\right)}{\left(y_{0}-\varepsilon_{y_{0}}\right)}\left(a+\varepsilon_{a}\right)^{4}\right]
\end{aligned}
$$

If the measurement of displacement is taken at only, for example the middle point of the beam, then we can not prognose the parameters $a, b, h, E$. 
Indeed we only have a equation

$$
y_{0}=\frac{5 q a^{2}}{384 b h^{3} E}
$$

of four unknowns $E, h, b, a$.

2. Case of a circular plate simply supported edge subjected to a uniformly distributed load $q$, the radius of the plate is a (Fig. 3). We have

$$
W_{\max }=W(r=0)=\frac{5+\mu}{1+\mu} \cdot \frac{q a^{4}}{64 D}
$$

then

$$
D=\frac{5+\mu}{64(1+\mu)} q a^{4} \cdot \frac{1}{W_{\max }}
$$

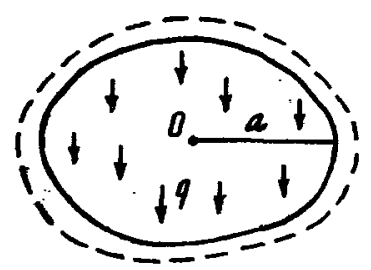

Fig. 3

Obviously if we have $W_{\max }, q, a, \mu$ then we got $D$, but

$$
D=\frac{E h^{3}}{12\left(1-\mu^{2}\right)} \Rightarrow E=\frac{12\left(1-\mu^{2}\right) D}{h^{3}}
$$

Note that in this example, if we change $q$ to get different $W_{\max }$ then we'll obtain only similar informations.

3. Diagnosis of the characteristics of material of a plate via measurement data of displacements [8].

Step 1. The task of diagnosis. To find $E, E J$ or $D$.

Step 2. Let the displacements are measured at a number of points.

$$
\vec{U}^{0}=\left\{U_{1}^{(0)}, U_{2}^{(0)}, \ldots, U_{m}^{(0)}\right\}
$$

Note that while measuring displacements, only the displacements produced strains, whereas the hard displacements (hold displacements) are deleted.

Step 3.

a. The diagnosis equations are the equations of the finite element method, in which variables are displacements, the diagnosis unknown is $E$ (moduli of elastic material). 
We can prognosis $a \leq E \leq 0$, we discretize $E$ by set

$$
E=\left\{a<E_{1}<E_{2}<\ldots<E_{p}<b\right\} .
$$

b. With $E=E_{j}(j=1,2, \ldots, p)$, making use of the program to compute the decign problem, we obtain the displacements of the structure.

$$
\left\{U_{1}^{(j)}, U_{2}^{(j)}, \ldots, U_{N}^{(j)}\right\}
$$

c. Find the dispersions $\Delta_{j}$

$$
\Delta_{j}=\left\{\sum_{i=1}^{m}\left(U_{i}^{(j)}-U_{i}^{(0)}\right)^{2}\right\}^{\frac{1}{2}} .
$$

d. Find

$$
\Delta_{s}=\min _{(j)} \Delta_{j}
$$

Example: Consider a rectangular plate simply supported at corners (Fig. 4) subjected to a uniformly distributed load $q=2.4 \mathrm{KN} / \mathrm{m}^{2}$, concentrated force $P=20 K N$ at the middle point of plate, thickness of plate $h=8 \mathrm{~cm}$, Poisson's coefficient $\mu=0.23$.

\begin{tabular}{|c|c|c|c|}
\hline Nodal & $U_{1}^{(0)}(\mathrm{cm})$ & Nodal & $U_{1}^{(0)}(\mathrm{cm})$ \\
\hline 1 & 1.95 & 263 & 0.95 \\
\hline 13 & 1.34 & 275 & 0.18 \\
\hline 132 & 1.44 & 382 & 0.70 \\
\hline 144 & 0.68 & 394 & 0.46 \\
\hline 151 & 1.15 & 513 & 0.35 \\
\hline
\end{tabular}

- The task of diagnosis is to find moduli $E$.

- Let the displacements are measured be at ten nodal points (see table 1)

Table 1

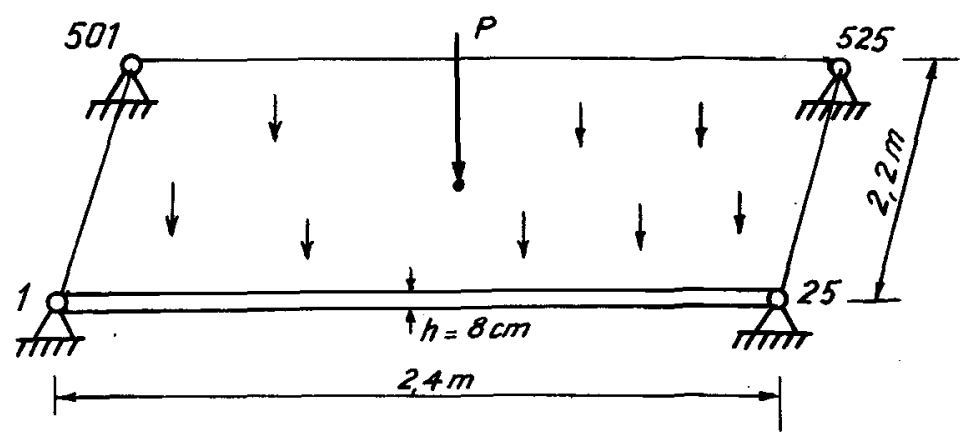

Fig. 4 
- Suppose that

$$
\begin{gathered}
E=\left\{1.8 \cdot 10^{7}<E_{1}<E_{2}<E_{3}<E_{4}<E_{5}<2.4 \cdot 10^{7}\right\} \\
E_{1}=1.8 \cdot 10^{7} \mathrm{KN} / \mathrm{m}^{2}, \quad E_{2}=1.9 \cdot 10^{7} \mathrm{KN} / \mathrm{m}^{2} \\
E_{3}=2.0 \cdot 10^{7} \mathrm{KN} / \mathrm{m}^{2}, \quad E_{4}=2.2 \cdot 10^{7} \mathrm{KN} / \mathrm{m}^{2} \\
E_{5}=2.4 \cdot 10^{7} \mathrm{KN} / \mathrm{m}^{2} . \text { It is according to concrete } \mathrm{M} \cdot 135-200 .
\end{gathered}
$$

The computed results on SAP90 with $E=1.8 \cdot 10^{7} \mathrm{KN} / \mathrm{m}^{2}$ is given the solution of design problem (see table 2) with $U_{1}^{(0)}=1.95 \mathrm{~cm}, U_{25}^{(0)}=U_{501}^{(0)}=0$ $\left.U_{525}^{(0)}=0\right)$.

$$
\begin{aligned}
& \Delta_{1}=\left[\sum_{i=1}^{10}\left(U_{i}^{(1)}-U_{i}^{(0)}\right)^{2}\right]^{\frac{1}{2}} \\
& \Delta_{1}=0,07640
\end{aligned}
$$

\begin{tabular}{|c|c|}
\hline$E\left(K N / m^{2}\right)$ & $\Delta_{i}$ \\
\hline$E_{1}=1.8 \cdot 10^{7}$ & 0.07650 \\
\hline$E_{2}=1.9 \cdot 10^{9}$ & 0.03132 \\
\hline$E_{3}=2.0 \cdot 10^{7}$ & 0.04767 \\
\hline$E_{4}=2.2 \cdot 10^{7}$ & 0.12403 \\
\hline$E_{5}=2.4 \cdot 10^{7}$ & 0.19512 \\
\hline
\end{tabular}

Other values of $E$ are computed similarly (see table 3)

Table 2

\begin{tabular}{ccccc}
\hline Nodal & & $U_{i}^{(0)}(\mathrm{cm})$ & $U_{i}^{(1)}(\mathrm{cm})$ & $\left(U_{i}^{(1)}-U_{i}^{(0)}\right)^{2}$ \\
\cline { 5 - 5 } 1 & 1.95 & & 1.95 & 0 \\
13 & 1.34 & & 1.356778 & 0.000282 \\
132 & 1.44 & & 1.450564 & 0.0001116 \\
114 & 0.68 & & 0.719314 & 0.001546 \\
251 & 1.15 & & 1.165324 & 0.000235 \\
263 & 0.95 & & 0.993874 & 0.001925 \\
275 & 0.18 & & 0.190324 & 0.000107 \\
382 & 0.70 & & 0.719314 & 0.000373 \\
294 & 0.46 & & 0.475564 & 0.000242 \\
513 & 0.35 & 0.381878 & 0,001016 \\
\hline
\end{tabular}

Table 9 
- Find $\min _{(i=1,2,3,4,5)} \Delta_{i}=\Delta_{2}=0.03123$, then $E_{a}=E_{2}=1.9 \cdot 10^{7} \mathrm{KN} / \mathrm{m}^{2}$

If value $\Delta_{0}$ is chosen, for example $\Delta_{0}=0.035$, then $\Delta_{2} \leq \Delta_{0}$ consequently $E=1.9 \cdot 10^{7} \mathrm{KN} / \mathrm{m}^{2}$.

\section{Conclusions}

1. The diagnosis depends on three main parts:

- The modeling real construction

- Measurement's data

- The algorithm for diagnosis.

2. In this paper the author mainly presented an algorithm of technical diagnosis, other parts have been discussed preliminary [9].

3. Up to now, there is no a general algorithm for diagnosis problem. The method proposed above can use for a large class of technical diagnosis problems of construction, in addition which can use for economical system, ecological system, and social system.

This publication is completed with financial support from the Council for Natural Sciences of Vietnam.

\section{REFERENCES}

1. Bui Huy Duong. Inverse problem in material mechanics, (in Vietnamesetranslated from French by Nguyen Dong Anh) Construction Publisher, Hanoi 1996.

2. A. N. Chikhonov, V. Ia. Arxenhin. Methods for solving incorrect problems. Nauka. Moscow 1979 (in Russian).

3. Nguyen Van Pho. Le Ngoc Hong and Le Ngoc Thach. On the numerical methods for solving technical diagnosis problem. Proceedings of the sixth national congress on Mechanics, Hanoi 1997 (in Vietnamese).

4. Nguyen Cao Menh, Nguyen Tien Khiem, Do Son, Dao Nhu Mai, Nguyen Viet Khoa. Procedure for diagnosis of fixed offshore constructions by dynamic characteristics. Proceeding of the fifth national conference on Solid Mechanics, Hanoi 1996 (in Vietnamese).

5. Bui Duc Chinh. Model for safety evaluation of reinforced concrete bridges based upon the results of inspection and field testing. Proceedings of the sixth national congress on Mechanics, Hanoi 1997 (in Vietnamese).

6. Zienkiewicz O. C., Taylor R. L. The finite element Method Mc. Graw-Hill book company 1989. 
7. Fedorov V. V. Optimum experimental theory "Nauka" Moscow 1971 (in Rusian).

8. Pham Van Thiet. A method for solving diagnosis problem via measurement's data of displacements. Thesis of master degree. Hanoi University of Civil Engineering 1988 (in Vietnamese).

9. Nguyen Van Pho. Application of reliability theory into stochastic stability problem. Proceedings of the sixth national congress on Mechanics - Hanoi 1997 (in Vietnameses).

Received January 6, 1999

MộT PHUƠNG PHÁP CHẨN ĐOÁN KỸ THUẬT CÔNG TRÌNH

Trong bài này tác giả đề nghị một phương pháp chẩn đoán kỹ thuật cho hệ thống nói chung và công trình nói riêng.

Thuật toán chẩn đoán là một quá trình lặp, giải một dãy các bài toán thuận (bài toán thiết kế), kết hợp với ý tưởng giải bài toán tối ưu đã được dùng trong lý thuyết thực nghiệm tối ưu, để nhanh chóng tìm ra lời giải bài toán ngược (bài toán chẩn đoán). Nhờ vậy đã tránh được các khó khăn khi giải bài toán ngược của cơ học công trình.

Đã dùng SAP90 để chẩn đoán môđun đàn hồi $E$ của tấm bê tông chữ nhật, chịu tải phân bố đều và tập trung có lún lệch ̛ơ một góc. 